TECHNICAL NOTE

\author{
D.L. Cooke \\ M. Levitt \\ L.J. Kim \\ D.K. Hallam \\ B. Ghodke
}

\section{Transcranial Access Using Fluoroscopic Flat Panel Detector CT Navigation}

SUMMARY: FPCT and navigation software on contemporary fluoroscopic units perform imaging of a quality comparable with conventional CT. They can accurately guide percutaneous procedures, providing live instrument visualization and the capability to re-image without patient transfer. FPCT navigation was used in the placement of a ventricular drain in a 62-year-old woman for subarachnoidrelated hydrocephalus by using an otherwise standard bedside technique. Ventriculostomy catheter placement was technically successful without complication with a catheter at the foramen of Monro.

ABBREVIATIONS: DSA = digital subtraction angiography; FPCT $=$ flat panel detector $\mathrm{CT}$
$\mathbf{F}_{\mathrm{t}, \mathrm{s}}$ PCT and integrated guidance software available on contemporary fluoroscopic systems emit a lower radiation dose while producing imaging of a quality comparable with that of conventional CT. ${ }^{1-5}$ They also have the ability to perform percutaneous procedures within the body, spine, and head and neck safely and accurately. ${ }^{4-11}$ FPCT demonstrates excellent spatial resolution and, though limited in subtle contrast differentiation, is of diagnostic accuracy in the assessment of general cerebral anatomy and higher attenuation features such as hemorrhage. ${ }^{5-8,10,11}$ Beyond the ability of FPCTbased navigation to quickly and accurately target a particular focus is the provision of real-time instrument visualization using live fluoroscopic overlay and the capability of re-imaging without moving the patient, limitations of current neuronavigational programs. ${ }^{10}$

Ventricular drain catheter placement in the setting of subarachnoid hemorrhage-related hydrocephalus is a common neurosurgical procedure. Performed at the bedside by using anatomic landmarks, ventriculostomy placement is safe and accurate with a low rate of complications. ${ }^{12-15}$ Given the frequent need for ventricular drain placement and the relative technical ease of the procedure, we chose it as means to demonstrate proof of principle regarding transcranial FPCT guidance. We herein describe the use of the navigation system in the placement of a ventriculostomy catheter.

\section{Case Report}

A 62-year-old woman presented with altered mental status. Physical examination revealed an intubated obtunded patient with left-sided hemiplegia and equal and reactive pupils. CT of the head showed diffuse subarachnoid hemorrhage, hydrocephalus, and significant hemorrhage in the basal cisterns. CT angiography revealed a $6 \times 7$ $\mathrm{mm}$ right-sided posterior communicating artery aneurysm and a $3 \times$ $2 \mathrm{~mm}$ left-sided middle cerebral artery aneurysm.

The patient underwent emergent FPCT-guided ventriculostomy placement in the angiography suite. To minimize movement, we carefully taped her head to the table. The left scalp was prepped, and a

Received December 4, 2009; accepted January 10, 2010.

From the Departments of Radiology (D.L.C., D.K.H., B.G.), and Neurological Surgery (M.L., L.J.K., D.K.H., B.G.), University of Washington School of Medicine, Seattle, Washington. Please address correspondence to Daniel L. Cooke, MD, University of Washington School of Medicine, 1959 NE Pacific St, NW011, Box 357115, Seattle, WA 98195-7115; e-mail: dcooke@uw.edu

Indicates article with supplemental online video.

DOI 10.3174/ajnr.A2066 $1-\mathrm{cm}$ incision was made at the Kocher point, approximately $11 \mathrm{~cm}$ posterior to the glabella and $3 \mathrm{~cm}$ lateral to midline in the midpupillary line. A twist-drill craniostomy was performed. The dura and pia mater were cauterized and incised.

Before catheter placement, an FPCT (XperCT and XperGuide; Philips Healthcare, Andover, Massachusetts) was performed and the data were analyzed on the 3D workstation. XperGuide analysis software was used to define the target as the ipsilateral foramen of Monro and the entry point at the craniostomy site. Using a progress view in the lateral plane, we passed a ventriculostomy catheter along the defined trajectory to a depth of $6.75 \mathrm{~cm}$, with the tip reaching the target at the foramen of Monro (Fig 1, on-line video). The surgeon was able to advance the catheter without prolonged fluoroscopic exposure and could stand comfortably adjacent to the C-arm. We noted that during catheter-guidance planning, the bull's eye or "entry" view generated by the navigational software often necessitated a working angle outside the range of the $\mathrm{C}$-arm when using a standard Kocher point entry site. To remedy this, we found that $10^{\circ}-15^{\circ}$ of head flexion or the reverse Trendelenburg position was adequate to address this problem.

CSF was noted to be under high pressure. The catheter was secured and the incision was closed. A second FPCT was then performed, confirming accurate catheter placement, which was also shown on a subsequent formal head CT (Fig 2). The ventriculostomy catheter tip terminated in the expected location at the foramen of Monro, and no placement-related complications were noted on post-head CT. The procedure required 12.3 minutes of fluoroscopy and produced $273 \mathrm{~Gy} / \mathrm{cm}^{2}$ of dose area product. Overall, guidance added approximately 30-40 minutes to the traditional bedside technique. The patient then underwent diagnostic cerebral angiography and eventual balloon-assisted coiling.

\section{Discussion}

To the authors' knowledge, this is the first report of the use of FPCT navigation for transcranial access. There are reports documenting the use of standard CT and virtual navigation systems for the placement of external ventricular catheters, though neither provides the ability to immediately re-image the patient while also providing real-time visualization during catheter placement. $^{16,17}$

The main limitation of FPCT navigation is the need for the patient to remain stationary during the procedure. Most neurosurgical guidance systems necessitate a Mayfield clamp to immobilize the head, a device not currently compatible with a conventional angiography table. In the setting of an FPCT- 


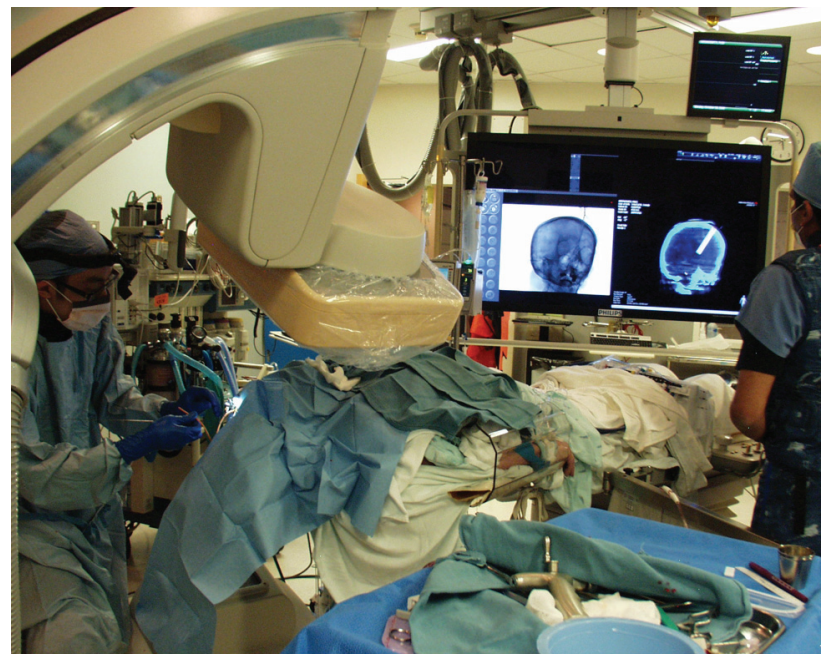

Fig 1. Ventricular drain placement. The surgeon is working adjacent to the C-arm during placement of the ventriculostomy catheter.

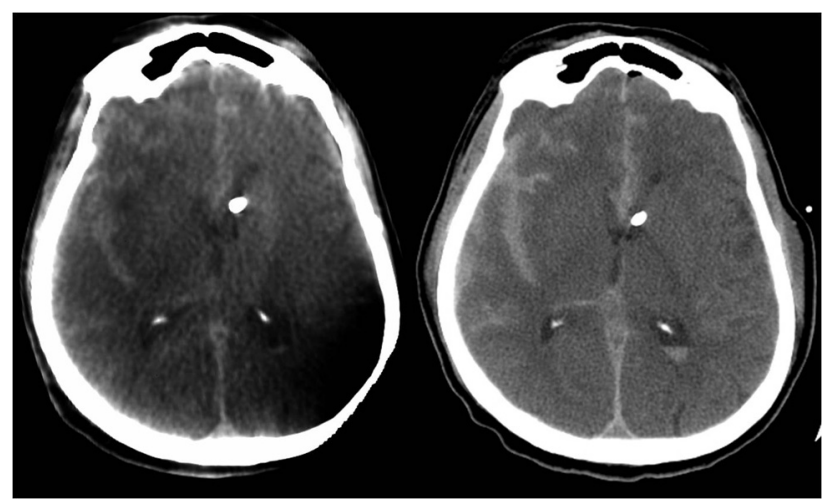

Fig 2. Axial images from a FPCT (left) and a standard noncontrast CT (right) demonstrate the tip of the ventricular catheter at the foramen of Monro.

guided procedure, securely taping a patient's head and the use of general anesthesia help minimize potential patient motion, though not to the degree provided by a true surgical clamp. In light of such a limitation, the FPCT guidance system has the ability to reorient the CT image onto live fluoroscopy by using the patient's osseous anatomy as reference landmarks, much in the manner that fiducials are used in other neuro-navigation systems. ${ }^{10}$ Ultimately, if there is concern that the patient or the intracranial anatomy has moved significantly, a new FPCT and trajectory can be acquired without transferring the patient.

The use of FPCT navigation will not likely supplant the traditional placement of ventricular drains, given the increase in time and cost, though it could prove helpful in those patients with slit-like ventricles or significantly distorted intracranial anatomy. With optimization, such a navigational system could be used for tissue resection (therapeutic and diagnostic in nature), general fluid aspiration (CSF, abscess, hemorrhage), and drug or device delivery for any number of neurologic pathologies. For example, there is evidence demonstrating the safety and technical success of frameless stereo- tactic intracerebral hemorrhagic aspiration. ${ }^{18-21}$ The evacuation of deep intracerebral hemorrhage requiring conventional neuro-navigational software could be further optimized by using FPCT navigation techniques. Additionally, the ability to simultaneously perform endovascular intervention opens the door to true tandem procedures, (eg, stereotactic aspiration of an epidural hematoma and meningeal artery embolization).

\section{References}

1. Braak S, van Strijen M, Van Leersum M, et al. Effective dose during needle interventions: real time 3D fluoroscopy guidance versus CT fluoroscopy. In: Proceedings of the 94th Scientific Assembly and Annual Meeting of the Radiological Society of North America, Chicago, Illinois. November 30-December 5, 2008

2. Racadio J, Yoshizumi T, Toncheva G, et al. Radiation dosimetry evaluation of $\mathrm{C}$-arm cone beam CT for pediatric interventional radiology procedures: a comparison with MDCT. In: Proceedings of the 94th Scientific Assembly and Annual Meeting of the Radiological Society of North America, Chicago, Illinois. November 30-December 5, 2008

3. Noordhoek NJ, van de Haar P, Timmer J. Direct comparison of commercially available C-arm CT to multi-slice CT image quality. In: Proceedings of the 94th Scientific Assembly and Annual Meeting of the Radiological Society of North America, Chicago, Illinois. November 30-December 5, 2008

4. Miracle A, Mukherji S. Conebeam CT of the head and neck. Part 1. Physical principles. AJNR Am J Neuroradiol 2009;30:1088-95. Epub 2009 May 13

5. Miracle A, Mukherji S. Conebeam CT of the head and neck. Part 2. Clinical applications. AJNR Am J Neuroradiol 2009;30:1285-92. Epub 2009 May 20

6. Heran N, Song J, Namba K, et al. The utility of DynaCT in neuroendovascular procedures. AJNR Am J Neuroradiol 2006;27:330-32

7. Daly M, Siewerdsen J, Moseley D, et al. Intraoperative cone-beam CT for guidance of head and neck surgery: assessment of dose and image quality using a C-arm prototype. Med Phys 2006;33:3767-80

8. Doelken M, Struffert T, Richter G, et al. Flat-panel detector volumetric CT for visualization of subarachnoid hemorrhage and ventricles: preliminary results compared to conventional CT. Neuroradiology 2008;50:517-23

9. Söderman M, Babic D, Holmin S, et al. Brain imaging with a flat detector C-arm: technique and clinical interest of XperCT. Neuroradiology 2008;50: 863-68. Epub 2008 Jun 17

10. Racadio J, Babic D, Homan R, et al. Live 3D guidance in the interventional radiology suite. AJR Am J Roentgenol 2007;189:W357-64

11. Dörfler A, Struffert T, Engelhorn T, et al. Rotational flat-panel computed tomography in diagnostic and interventional neuroradiology. Rofo 2008;180: 891-98

12. Huyette D, Turnbow B, Kaufman C, et al. Accuracy of the freehand pass technique for ventriculostomy catheter placement: retrospective assessment using computed tomography scans. J Neurosurg 2008;108:88-91

13. Binz DD, Toussaint LG 3rd, Friedman JA. Hemorrhagic complications of ventriculostomy placement: a meta-analysis. Neurocrit Care 2009;10:253-56. Epub 2009 Feb 18

14. Kakarla UL, Kim LJ, Chang SW, et al. Safety and accuracy of bedside external ventricular drain placement. Neurosurgery 2008;63(1 suppl 1):ONS162-66, discussion ONS166-67

15. Saladino A, White J, Wijdicks E, et al. Malplacement of ventricular catheters by neurosurgeons: a single institution experience. Neurocrit Care 2009;10:248-52

16. Banerjee P, Luciano C, Lemole GJ, et al. Accuracy of ventriculostomy catheter placement using a head- and hand-tracked high-resolution virtual reality simulator with haptic feedback. J Neurosurg 2007;107:515-21

17. Krötz M, Linsenmaier U, Kanz K, et al. Evaluation of minimally invasive percutaneous CT-controlled ventriculostomy in patients with severe head trauma. Eur Radiol 2004;14:227-33

18. Barrett R, Hussain R, Coplin W, et al. Frameless stereotactic aspiration and thrombolysis of spontaneous intracerebral hemorrhage. Neurocrit Care 2005;3:237-45

19. Morgan T, Zuccarello M, Narayan R, et al. Preliminary findings of the minimally-invasive surgery plus rtPA for intracerebral hemorrhage evacuation (MISTIE) clinical trial. Acta Neurochir Suppl 2008;105:147-51

20. Vespa P, McArthur D, Miller C, et al. Frameless stereotactic aspiration and thrombolysis of deep intracerebral hemorrhage is associated with reduction of hemorrhage volume and neurological improvement. Neurocrit Care 2005;2:274-81

21. Thiex R, Rohde V, Rohde I, et al. Frame-based and frameless stereotactic hematoma puncture and subsequent fibrinolytic therapy for the treatment of spontaneous intracerebral hemorrhage. J Neurol 2004;251:1443-50 